\title{
Density Propagator for Many-Body Localization: Finite-Size Effects, Transient Subdiffusion, and Exponential Decay
}

\author{
Soumya Bera, ${ }^{1,2}$ Giuseppe De Tomasi, ${ }^{2}$ Felix Weiner, ${ }^{3}$ and Ferdinand Evers ${ }^{3}$ \\ ${ }^{1}$ Department of Physics, Indian Institute of Technology Bombay, Mumbai 400076, India \\ ${ }^{2}$ Max-Planck-Institut für Physik komplexer Systeme, Nöthnitzer Straße 38, 01187-Dresden, Germany \\ ${ }^{3}$ Institute of Theoretical Physics, University of Regensburg, D-93050 Regensburg, Germany \\ (Received 17 October 2016; revised manuscript received 30 January 2017; published 8 May 2017)
}

\begin{abstract}
We investigate charge relaxation in quantum wires of spinless disordered fermions ( $t-V$ model). Our observable is the time-dependent density propagator $\Pi_{\varepsilon}(x, t)$, calculated in windows of different energy density $\varepsilon$ of the many-body Hamiltonian and at different disorder strengths $W$, not exceeding the critical value $W_{c}$. The width $\Delta x_{\varepsilon}(t)$ of $\Pi_{\varepsilon}(x, t)$ exhibits a behavior $d \ln \Delta x_{\varepsilon}(t) / d \ln t=\beta_{\varepsilon}(t)$, where the exponent function $\beta_{\varepsilon}(t) \lesssim 1 / 2$ is seen to depend strongly on $L$ at all investigated parameter combinations. (i) We confirm the existence of a region in phase space that exhibits subdiffusive dynamics in the sense that $\beta_{\varepsilon}(t)<1 / 2$ in a large window of times. However, subdiffusion might possibly be transient, only, finally giving way to a conventional diffusive behavior with $\beta_{\varepsilon}=1 / 2$. (ii) We cannot confirm the existence of many-body mobility edges even in regions of the phase diagram that have been reported to be deep in the delocalized phase. (iii) (Transient) subdiffusion $0<\beta_{\varepsilon}(t) \lesssim 1 / 2$ coexists with an enhanced probability for returning to the origin $\Pi_{\varepsilon}(0, t)$, decaying much slower than $1 / \Delta x_{\varepsilon}(t)$. Correspondingly, the spatial decay of $\Pi_{\varepsilon}(x, t)$ is far from Gaussian, being exponential or even slower. On a phenomenological level, our findings are broadly consistent with the effects of strong disorder and (fractal) Griffiths regions.
\end{abstract}

DOI: 10.1103/PhysRevLett.118.196801

Introduction.-The discovery of many-body localization (MBL) has attracted considerable attention over recent years and gave rise to a new research field [1-5]. An analytical proof of MBL has been given with minimal assumptions in spin chains with random local interactions [6]. Such MBL phases are characterized by the absence of transport and thermalization [7-10], which has been attributed to a set of quasilocal integrals of motion [11-14]. Anticipating that these integrals of motion adiabatically connect to their noninteracting analogues, it is perhaps natural to assume that there should be an adiabatic connection between localized eigenstates as well $[6,15]$.

The MBL phase is distinguished from another phase that exhibits a degree of delocalization and which therefore is believed to be (thermal) ergodic [7,16,17]. The corresponding relaxation dynamics may not, however, reflect the simple diffusive behavior familiar from conventional metals. Instead, a subdiffusive scaling of the (spin-) density correlations has been reported [18-23] (though some studies concluded differently [24,25]). It was understood to indicate Griffiths effects [19,26-28] near the MBL transition. Interestingly, it has been proposed that different behavior within these phases may also exist that exhibit diffusive relaxation of one conserved quantity (charge, energy, or spin) and a subdiffusive behavior in another $[23,29]$. Clearly, a coexistence of localized and delocalized behavior would be incompatible with generic expectations based on conventional mode-coupling ideas [30].

The phase transition between the MBL phase and the delocalized phase is not yet well understood. For instance, it has been shown that at very large values of the disorder $W$ all eigenstates of the many-body Hamiltonian $\hat{H}(W)$ are localized [7,9,31-33], while with disorder dropping below a critical value $W<W_{c}$ a transition could occur below which $\hat{H}(W)$ supports a delocalized spectral density window [9,32,34-38]; see Fig. 1. At present, the width of this window is a matter of controversy. Recent numerical works on the random-field Heisenberg chain [9], the disordered Ising chain [32], and recent work on the Aubry-André model [39] were interpreted as giving evidence for the existence of a many-body mobility edge

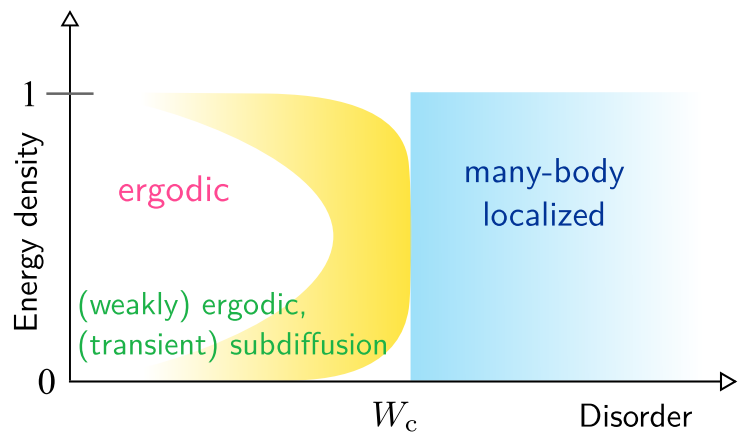

FIG. 1. A qualitative phase diagram of different dynamical regions in the disorder energy-density plane of the $t-V$ model. At disorder strength $W$ below the many-body localization transition $W_{c}$, we propose a transient subdiffusive, weakly ergodic dynamical regime with an anomalously slow decay of the return probability. 
(MBME) that separates a band of delocalized states from localized band edges. Later authors have argued, however, that results can be significantly contaminated with finite size effects unless carefully extrapolated. For instance, the phase boundary as found in Ref. [9] should be shifted to large disorder values as argued in Ref. [40]. In fact, the very existence of MBME was called into question by De Roeck et al., who suggested that the presence of a delocalized spectral window should imply the possibility for the formation of hot bubbles of electronic liquid that destabilize localizing processes in all spectral density windows [41].

In this work, we investigate the charge propagation focusing on the delocalized region near the MBL transition. A common description of relaxation dynamics employs the density propagator $\Pi(x, t)$ that takes a simple Gaussian shape for diffusive systems: $\Pi(x, t)=e^{-\frac{1}{2}[x / \Delta x(t)]^{2}} /$ $\sqrt{2 \pi} \Delta x(t), \Delta x(t)=\sqrt{D t}$, where $D$ is the diffusion constant. Aiming at mobility edges, we actually study a variant of it, $\Pi_{\varepsilon}(x, t)$, that resolves the contribution to $\Pi(x, t)$ stemming from many-body states with energy densities $\varepsilon$. We thus get access to the length scales relevant for the crossover physics, which allow us to carefully monitor finite size and finite time effects. In this way we go beyond previous studies.

We outline our results. (i) Within our observation window, $\Pi_{\varepsilon}(x, t)$ exhibits a very pronounced nonGaussian spatial shape that decays in a (simple) exponential fashion or even slower. It is tempting to associate this finding with the stretched exponential behavior of correlations that has recently been proposed to exist due to fractal Griffiths regions in the localized phase near the phase boundary [42]. (ii) Because of this peculiar shape of $\Pi_{\varepsilon}(x, t)$, the time dependence of its width $\Delta x_{\varepsilon}(t)$ is very sensitive to the system size, $L$. In order to highlight the effects of finite size in the time evolution, we investigate the exponent scaling function

$$
\beta_{\varepsilon}(t) \equiv \frac{d \log \Delta x_{\varepsilon}(t)}{d \log t},
$$

which at long times quantifies the rate of growth of $\Delta x_{\varepsilon}(t) \propto t^{\beta_{\varepsilon}(t=\infty)}$, and for diffusive systems $\beta_{\varepsilon}=1 / 2$. In the ergodic phase at intermediate times $\beta_{\varepsilon}(t)$ grows in a subdiffusive manner with values $\beta_{\varepsilon}(t)<1 / 2$ consistent with the earlier reports [18,19,21-23]. However with increasing time, $\beta_{\varepsilon}(t)$ becomes progressively $L$ dependent. At these longer times a similar tendency of growing $\beta_{\varepsilon}(t)$ (with $L$ ) is observed in all spectral windows-at low, intermediate, and high energy density. This strong growths prevents us from confirming the existence of genuine subdiffusion that would exhibit a time-independent exponent $\beta_{\varepsilon}<1 / 2$. We detect a slow growth of $\beta_{\varepsilon}(t)$ even in those regions of the phase diagram that have been identified previously as localized. Thus, the (delocalized) phase is larger than reported previously, which is associated with a very slow collective dynamics [43].

(iii) For the probability $\Pi_{\varepsilon}(0, t)$ to return to the origin one might have suspected $\Pi_{\varepsilon}(0, t) \propto 1 / \Delta x_{\varepsilon}(t)$, suggesting $\Pi_{\varepsilon}(0, t) \propto t^{-\beta_{\varepsilon}(t=\infty)}$. Instead, our data indicate that the subdiffusive transients coexist with an elevated return probability consistent with (possibly transient) weakly ergodic subphases with fractal phenomenology, $\Pi_{\varepsilon}(0, t) \propto$ $\Delta x_{\varepsilon}(t)^{-\alpha_{\varepsilon}}$ and $0 \leq \alpha_{\varepsilon}<1$.

Model and method.-Like several works before $[9,17,18,34,35,44-46]$, we consider the $t$ - $V$ model

$$
\begin{aligned}
\hat{\mathcal{H}}= & -\frac{t_{h}}{2} \sum_{x=-L / 2}^{L / 2-2} \hat{c}_{x}^{\dagger} \hat{c}_{x+1}+\text { H.c. }+\sum_{x=-L / 2}^{L / 2-1} \mu_{x}\left(\hat{n}_{x}-\frac{1}{2}\right) \\
& +V \sum_{x=-L / 2}^{L / 2-2}\left(\hat{n}_{x}-\frac{1}{2}\right)\left(\hat{n}_{x+1}-\frac{1}{2}\right),
\end{aligned}
$$

where the summations are along an $L$-site wire, $x=1, \ldots, L$, with hopping $\left(t_{h}=1\right)$ and interaction $(V)$ between nearest neighbors, only; the uncorrelated on-site energies $\mu_{x}$ are being drawn from a box distribution $[-W, W]$. We work at a half filling and with open boundary conditions. For $V=1.0$, the MBL transition is believed to be at $W_{c} \approx 3.5$ [9]. The specific correlator $\Pi_{\varepsilon}(x, t)$ that we are interested in has not yet been investigated; it is defined via its Fourier space representation [47]:

$$
\Pi_{\varepsilon}(q, t)=\overline{\Phi_{\varepsilon}(q, t) / \Phi_{\varepsilon}\left(q, t=0^{+}\right)},
$$

where the disorder average is denoted by the overline. $\Phi_{\varepsilon}(q, t)$ is the Fourier transform of the energy-projected density relaxation functions

$$
\Phi_{\varepsilon}(x, t)=\left[\left\langle\hat{n}_{x}(t) \hat{n}_{0}\right\rangle_{\varepsilon}-\left\langle\hat{n}_{x}\right\rangle_{\varepsilon}\left\langle\hat{n}_{0}\right\rangle_{\varepsilon}\right] \Theta(t) .
$$

The projection into a narrow spectral range near $\varepsilon$ is facilitated by taking the expectation value of an operator $\langle\hat{\mathcal{O}}\rangle_{\varepsilon}=\operatorname{Tr} \hat{\mathcal{O}} \hat{\rho}(\varepsilon)$ with

$$
\hat{\rho}(\varepsilon)=\mathcal{N}^{-1} \int_{\varepsilon-\Delta \varepsilon / 2}^{\varepsilon+\Delta \varepsilon / 2} d \varepsilon^{\prime} \sum_{\gamma}^{N}|\gamma\rangle \delta\left(\varepsilon_{\gamma}-\varepsilon^{\prime}\right)\langle\gamma|,
$$

where $|\gamma\rangle$ denotes the eigenstates of the Hamiltonian (2) with energy-density $\varepsilon_{\gamma}=\left(E_{\gamma}-E_{\min }\right) /\left(E_{\max }-E_{\min }\right)$, where $E_{\gamma}$ are the many-body energies and $E_{\max \text { min }}$ denote the extremal values of the energy spectrum. $N$ represents the number of states in the energy density window $\Delta \varepsilon$, and it is exponentially large in $L$. By definition, $\Pi_{\varepsilon}(q=0, t)=1$ and for a conventional diffusive system we have a Gaussian shape, $\Pi_{\varepsilon}(q, t)=\exp \left\{-\left[\Delta x_{\varepsilon}(t) q\right]^{2}\right\} \Theta(t)$, with $\Delta x_{\varepsilon}(t)=\sqrt{D_{\varepsilon} t}$. For the time evolution, Eq. (4), we employ a standard Chebyshev-polynomial propagation [48]; 

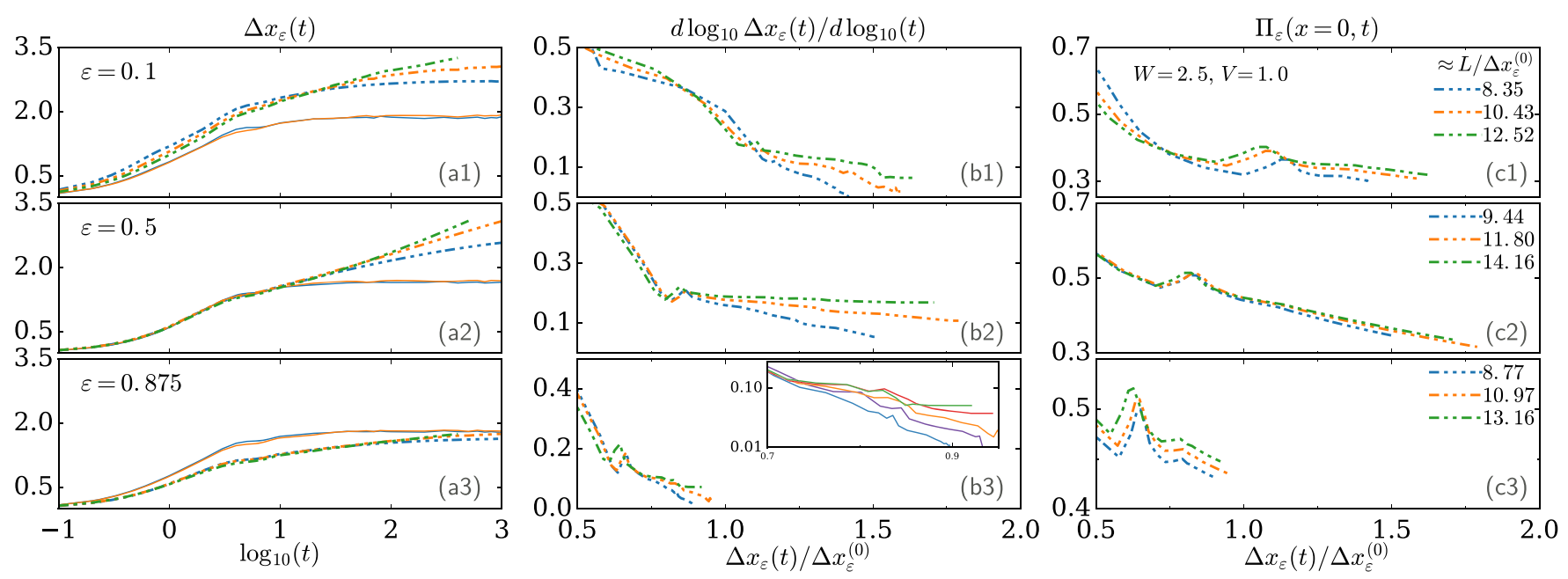

FIG. 2. (a1)-(a3) The time evolution of $\Delta x_{\varepsilon}(t)$ at $W=2.5$ and $V=1$ near the lower band edge (upper row, $\varepsilon=0.1$ ) in the center region (center row, 0.5) and near the upper band edge (lower row, 0.875) for system sizes $L=16,20,24$ (dashed traces blue, red, green). Also shown are noninteracting reference traces for $L=16,20\left(V=0\right.$, solid lines). (b1)-(b3) Replotting (a1)-(a3) as $d \ln \Delta x_{\varepsilon}(t) / d \ln t$ over $\Delta x_{\varepsilon}(t) / \Delta x_{\varepsilon}^{(0)}$ to highlight finite-size effects. Inset shows the enlargement of the (b3) data for better visibility of trends, including system sizes $L=16,18,20,22,24$ (bottom to top). (c1)-(c3) Probability to return to the origin. The legends in this column also give the three system sizes in units of the bare localization length. (In all calculations we fix the width of the energy window $\Delta \varepsilon=0.1$ [49]).

traces over operators are performed stochastically as averages over random state vectors. The approach owes its efficiency to the fact that disorder averages converge very rapidly with the number of random states. Details of the calculations and performance tests we relegate to the Supplemental Material [49].

Results.-We begin the analysis of the propagator $\Pi_{\varepsilon}(x, t)$ with its second moment in real space,

$$
\Delta x_{\varepsilon}(t)^{2}=\left\langle x^{2}\right\rangle_{\varepsilon}-\langle x\rangle_{\varepsilon}^{2}, \quad\left\langle x^{n}\right\rangle_{\varepsilon}=\sum_{x=-L / 2}^{L / 2-1} x^{n} \Pi_{\varepsilon}(x, t) .
$$

Figures 2(a1)-2(a3) show the $\Delta x_{\varepsilon}(t)$ at $W=2.5$ for both the interacting $(V=1$, dashed line) and noninteracting ( $V=0$, solid line) case for several values of energy densities $(\varepsilon=0.1,0.5,0.875)$. For these parameters MBMEs have been reported near $\varepsilon \approx 0.2$ and near 0.8 with a delocalized regime in between [9].

Figures 2(a1)-2(a3) carry several messages. (i) Finite size effects are very strong: the system size $L$ exceeds the noninteracting standard deviation $\Delta x_{\varepsilon}^{(0)}$ (saturation value in time), by a factor of $10-15\left(\approx L / \Delta x_{\varepsilon}^{(0)}\right)$, but nevertheless the growth of $\Delta x_{\varepsilon}(t)$ changes with $L$ by as much as $30 \%$. (ii) The interaction mediated delocalization process is very slow. Even after a time that typically corresponds to $0.1 \%$ of the inverse hopping $t_{h}^{-1}$ the width of the wave packet has grown by less than a factor of 2 as compared to $\Delta x_{\varepsilon}^{(0)}$. (iii) Depending on the spectral window, the transient dynamics is quite different. In particular, the spreading of $\Pi_{\varepsilon}(x, t)$ is enhanced by the interactions at low energy densities while it is hindered at high densities as compared to the noninteracting reference case.
Flowing exponent.- $\beta_{\varepsilon}(t)$.- To quantify the time dependence of $\Delta x_{\varepsilon}(t)$, we study the $\beta_{\varepsilon}(t)$ as defined in Eq. (1). Figures 2(b1)-2(b3) show the $\beta_{\varepsilon}$ function as a function of $\Delta x_{\varepsilon}(t) / \Delta x_{\varepsilon}^{(0)}$. It very clearly highlights the fact that beyond a certain transient time $\tau_{\varepsilon}$ (set by the kink position) a slow dynamics sets in which reveals itself by a high degree of sensitivity to the system size $L$. Moreover, as is seen in Figs. 2(b1)-2(b3) all traces of $\beta_{\varepsilon}(t)$ experience a kink with a position evolving with the energy density $\varepsilon$ that does not collapse after rescaling of the abscissa with $\Delta x_{\varepsilon}^{(0)}$.

While the range of $L$ values available to us is not sufficient to study the asymptotic limit (in $L$ and $t$ ), our data nevertheless give a nonvanishing lower bound for $\beta_{\varepsilon}(t)$ and, hence, indicate delocalization, at least near the band center. With this caveat, we notice that the qualitative behavior seen in all energy ranges is the same: With $L$ increasing, there is a pronounced trend for $\beta_{\varepsilon}(t)$ to grow (at fixed long time), see Figs. 2(b1)-2(b3) and inset. Strictly speaking, we thus find no evidence for an upper bound to $\beta_{\varepsilon}$ below the diffusion limit $1 / 2$, i.e., for genuine subdiffusion. Moreover, the growth (with $L$ ) being similar in all energy windows, we also find no evidence for the existence of a many-body mobility edge at $W=2.5$. The picture is similar for other choices of $W(\lesssim 3.0)$ [49]. At larger disorder and close to the transition, $W \approx W_{c}$, the situation is numerically less conclusive due to residual statistical noise. To account for this in Fig. 1, this region of the phase diagram has been left uncolored (white).

Return probability.- $\Pi_{\varepsilon}(x=0, t)$. - In one-dimensional diffusive systems the return probability associated with a spreading wave packet relates to the variance $\Pi_{\varepsilon}(0, t) \sim$ $1 / \Delta x_{\varepsilon}(t)$, merely stating that the wave packet is internally 


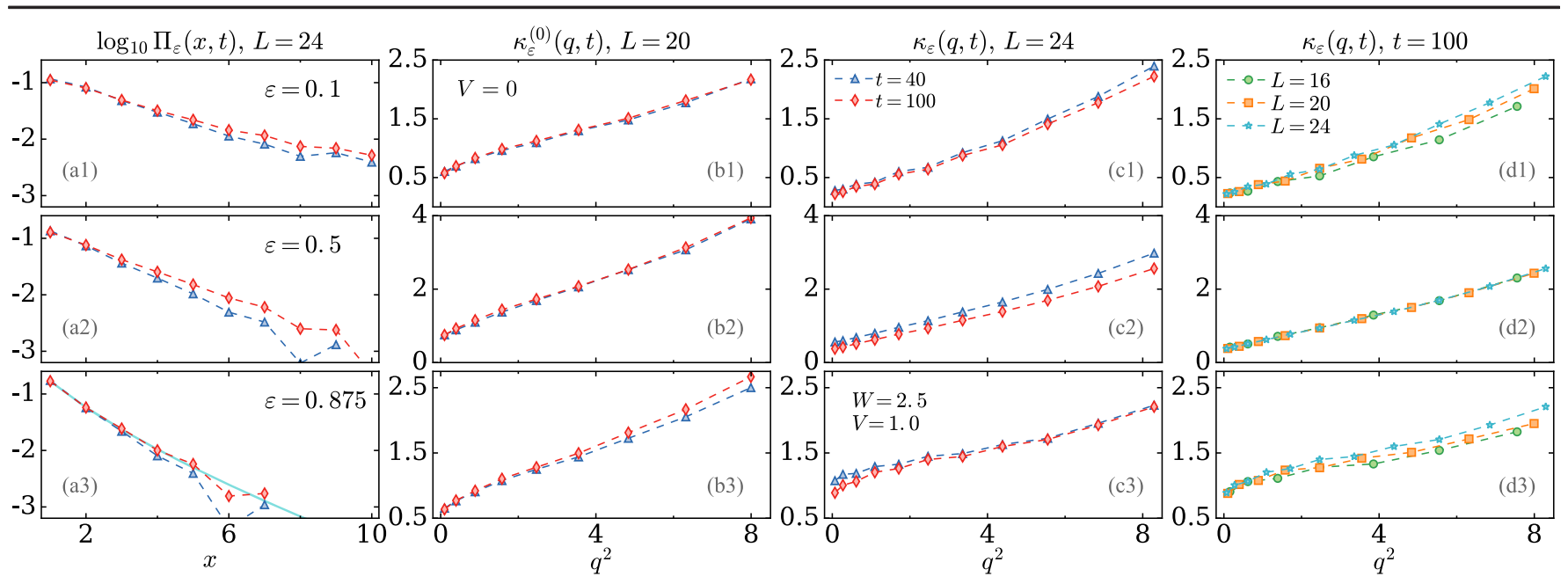

FIG. 3. (a1)-(a3) The density propagator $\Pi_{\varepsilon}(x, t)$ in the delocalized regime $(\varepsilon=0.1,0.5,0.875, W=2.5, L=24)$ at two times $t=40,100$. The log-normal plot illustrates non-Gaussian shape. Solid line in (a3) shows a stretched exponential fit with an exponent $\approx 0.7$. (b1)-(d3) The corresponding memory kernel $\kappa_{\varepsilon}(q, t)=q^{2} /\left[\Pi_{\varepsilon}^{-1}(q, t)-1\right]$, see also Eq. (6), for the case without (b1)-(b3) and with interactions (c1)-(d3). The structure at larger wave numbers illustrates the (nonexponential) short-distance behavior. The absence of effects in time (and system size, not shown) highlights the localized character of the noninteracting kernel $\kappa_{\varepsilon}^{(0)}$. In contrast, the evolution of the interacting kernel is the hallmark of delocalization. (d1)-(d3) The $L$ dependence of $\kappa_{\varepsilon}(t)$.

homogeneous. The data displayed in Figs. 2(c1)-2(c3) do not adhere to this fundamental idea: $\Pi_{\varepsilon}(0, t)$ is close to stationary and therefore does not follow the $1 / \Delta x_{\varepsilon}$ law, most clearly seen in the low and high energy density regimes. This observation finds a natural explanation adopting the idea of strong disorder induced fractality. Indeed, it is well known that in the presence of (multi-)fractality the return probability can be enhanced, $\Pi_{\varepsilon}(0, t) \propto \Delta x_{\varepsilon}^{-\alpha_{\varepsilon}}$, with $0 \leq$ $\alpha_{\varepsilon}<1$ [50]. A very slowly decaying return probability can therefore also indicate a fractal-type behavior, i.e., $\alpha_{\varepsilon}$ being significantly smaller than unity. Unfortunately, it is very challenging to extract $\alpha_{\varepsilon}$ reliably from our data, because our observation window for $\Delta x_{\varepsilon}(t) / \Delta x_{\varepsilon}^{(0)}$ does not exceed a factor 2-3.

Density propagator. $-\Pi_{\varepsilon}(x, t)$.- - To understand the transient subdiffusive behavior further, here we look at the time dependence of the full distribution function $\Pi_{\varepsilon}(x, t)$ both in real and $q$ space. Figures 3(a1)-3(a3) displays a densitypropagator $\Pi_{\varepsilon}(x, t)$ that is far from Gaussian. To highlight its shape (curvature at small $q$, large $x$ ) we rewrite $\Pi_{\varepsilon}(q, t)$ employing an (inverse) memory kernel $\kappa_{\varepsilon}(q, t)$,

$$
\Pi_{\varepsilon}(q, t)=\left[1+q^{2} / \kappa_{\varepsilon}(q, t)\right]^{-1},
$$

where $-\left.\partial_{q}^{2} \Pi_{\varepsilon}(q, t)\right|_{q=0}=2 / \kappa_{\varepsilon}(0, t) \sim \Delta x_{\varepsilon}(t)^{2}$. A numerical example can be read off from Figs. 3(b) and 3(c). It displays $\kappa_{\varepsilon}$ at three different energy densities at intermediate disorder strength $W=2.5$. Notice that the noninteracting kernel, $\kappa_{\varepsilon}^{(0)}(q, t)$, is rapidly growing with wave number $q$ [see Figs. 3(b1)-3(b3)]. This behavior reflects the presence of a short-distance cutoff $a$, such as the lattice constant, terminating the long-distance, exponential tail.
It exists in a similar way also in the interacting kernels $\kappa_{\varepsilon}(q, t)$; see Figs. 3(c), 3(d) [51] [52].

Conclusions. - In this work, we have considered the full space-time structure of the spectrally resolved density correlator, $\Pi_{\varepsilon}(x, t)$, allowing us to monitor finite size effects. (i) The processes that are characteristic of delocalized behavior are very slow. Even at observation times of order $10^{3}$ (in units of inverse hopping $t_{h}^{-1}$ ), $\Pi_{\varepsilon}(x, t)$ has spread over little more than the noninteracting length $\Delta x_{\varepsilon}^{(0)}$. (ii) Although the system size exceeds $\Delta x_{\varepsilon}^{(0)}$ by a large factor, finite size effects are substantial, reflecting a spreading of $\Pi_{\varepsilon}(x, t)$ that is far from Gaussian, possibly (stretched) exponential in the tails.

Because of strong finite-size effects, the exponents $\beta_{\varepsilon}(t)$ that describe the spreading dynamics of the variance of the density propagator $d \ln \Delta x_{\varepsilon}(t) / d \ln t=\beta_{\varepsilon}(t)$ are hard to quantify reliably. We are able to provide a lower bound for $\beta_{\varepsilon}(t)$ suggesting the absence of many-body mobility gaps in the $t-V$ model at values of $W$ not too close to the transition region-apparently consistent with recent analytical arguments [41]. Since we cannot provide an upper bound for $\beta_{\varepsilon}(t)<1 / 2$, we cannot confirm the existence of genuine subdiffusive behavior in the asymptotic limit; a logically possible alternative is a transient behavior with an effectively growing exponent $\beta_{\varepsilon}(t)$ that gradually converges to the diffusion limit $1 / 2$. Together with transient subdiffusive behavior, we observe a drastically enhanced return probability, which could be interpreted as $\Pi_{\varepsilon}(0, t) \propto$ $\Delta x_{\varepsilon}^{-\alpha_{\varepsilon}}$ in accord with the assumptions of fractality induced by strong-disorder physics.

Based on these findings we propose the following scenario: There is a time scale $\tau_{\varepsilon}$ beyond which a slow 
dynamics kicks in together with diffusive behavior. Approaching the MBL transition from the delocalized side, this time scale diverges; simultaneously, $\beta_{\varepsilon}(t)$ at times $t \lesssim \tau_{\varepsilon}$ is rapidly decreasing, which might suggest a small value of $\beta_{\varepsilon}$ at the MBL transition. In this scenario, the critical fixed point would carry excited states that exhibit phenomenological features reminiscent of (strong) multifractality [53].

We conclude with two remarks relating our work to the most recent literature. (a) Consistent with our findings, also Serbyn, Papic, and Abanin observe very strong finite size effects in their study of the Thouless energy [54]. Like us, they interpret their results as indicating that the system sizes are too short for observing the asymptotic thermalized behavior. Unlike us, they go a step further proposing that the numerical data at small system sizes (below $L=20$ ) already reveal hydrodynamic properties of the critical fixed point, such as multifractality. This conclusion for us is difficult to draw, because one would expect system-size independent exponents in the critical window, which we do not observe. (b) Recent studies of Anderson localization of random regular graphs (RRG) reveal a slow flow with system size out of a (quasi-)multifractal into an ergodic regime $[55,56]$. When interpreting $\Delta x_{\varepsilon}(t)$ as an effective system size, then the transient subdiffusive behavior observed by us finds a natural interpretation within the RRG perspective.

Discussions with I. Gornyi, A. D. Mirlin, and D. Polyakov are gratefully acknowledged. S. B. and G. D. T. also thank J. H. Bardarson, M. Heyl and F. Pollmann for discussions. The project was supported by DFG under Projects No. EV30/7-1 and No. EV30/11-1. S. B. acknowledges support from the ERC starting Grant QUANTMATT NO. 679722.

[1] D. Basko, I. Aleiner, and B. Altshuler, Ann. Phys. (Amsterdam) 321, 1126 (2006).

[2] I. V. Gornyi, A. D. Mirlin, and D. G. Polyakov, Phys. Rev. Lett. 95, 206603 (2005).

[3] R. Nandkishore and D. A. Huse, Annu. Rev. Condens. Matter Phys. 6, 15 (2015).

[4] E. Altman and R. Vosk, Annu. Rev. Condens. Matter Phys. 6, 383 (2015).

[5] R. Vasseur and J. E. Moore, J. Stat. Mech. Theor. Exp. (2016) 064010.

[6] J. Z. Imbrie, J Stat. Phys. 163, 998 (2016).

[7] A. Pal and D. A. Huse, Phys. Rev. B 82, 174411 (2010).

[8] E. Canovi, D. Rossini, R. Fazio, G. E. Santoro, and A. Silva, Phys. Rev. B 83, 094431 (2011).

[9] D. J. Luitz, N. Laflorencie, and F. Alet, Phys. Rev. B 91, 081103 (2015).

[10] J. H. Bardarson, F. Pollmann, and J. E. Moore, Phys. Rev. Lett. 109, 017202 (2012).

[11] M. Serbyn, Z. Papić, and D. A. Abanin, Phys. Rev. Lett. 111, 127201 (2013).
[12] D. A. Huse, R. Nandkishore, and V. Oganesyan, Phys. Rev. B 90, 174202 (2014).

[13] A. Chandran, V. Khemani, C. R. Laumann, and S. L. Sondhi, Phys. Rev. B 89, 144201 (2014).

[14] V. Ros, M. Müller, and A. Scardicchio, Nucl. Phys. B891, 420 (2015).

[15] B. Bauer and C. Nayak, J. Stat. Mech. (2013) P09005.

[16] E. Canovi, D. Rossini, R. Fazio, G. E. Santoro, and A. Silva, New J. Phys. 14, 095020 (2012).

[17] A. D. Luca and A. Scardicchio, Europhys. Lett. 101, 37003 (2013).

[18] Y. Bar Lev, G. Cohen, and D. R. Reichman, Phys. Rev. Lett. 114, 100601 (2015).

[19] K. Agarwal, S. Gopalakrishnan, M. Knap, M. Müller, and E. Demler, Phys. Rev. Lett. 114, 160401 (2015).

[20] Y. B. Lev and D. R. Reichman, Europhys. Lett. 113, 46001 (2016).

[21] D. J. Luitz, N. Laflorencie, and F. Alet, Phys. Rev. B 93, 060201 (2016).

[22] I. Khait, S. Gazit, N. Y. Yao, and A. Auerbach, Phys. Rev. B 93, 224205 (2016).

[23] M. Žnidarič, A. Scardicchio, and V. K. Varma, Phys. Rev. Lett. 117, 040601 (2016).

[24] O. S. Barišić, J. Kokalj, I. Balog, and P. Prelovšek, Phys. Rev. B 94, 045126 (2016).

[25] R. Steinigeweg, J. Herbrych, F. Pollmann, and W. Brenig, Phys. Rev. B 94, 180401 (2016).

[26] R. Vosk, D. A. Huse, and E. Altman, Phys. Rev. X 5, 031032 (2015).

[27] S. Gopalakrishnan, M. Müller, V. Khemani, M. Knap, E. Demler, and D. A. Huse, Phys. Rev. B 92, 104202 (2015).

[28] A. C. Potter, R. Vasseur, and S. A. Parameswaran, Phys. Rev. X 5, 031033 (2015).

[29] F. P. J. G. Vipin Kerala Varma, A. Lerose, and A. Scardicchio, arXiv:1511.09144.

[30] S. Gopalakrishnan, K. Agarwal, E. A. Demler, D. A. Huse, and M. Knap, Phys. Rev. B 93, 134206 (2016).

[31] M. Žnidarič, T. Prosen, and P. Prelovšek, Phys. Rev. B 77, 064426 (2008).

[32] J. A. Kjäll, J. H. Bardarson, and F. Pollmann, Phys. Rev. Lett. 113, 107204 (2014).

[33] M. Serbyn, Z. Papić, and D. A. Abanin, Phys. Rev. X 5, 041047 (2015).

[34] V. Oganesyan and D. A. Huse, Phys. Rev. B 75, 155111 (2007).

[35] S. Bera, H. Schomerus, F. Heidrich-Meisner, and J. H. Bardarson, Phys. Rev. Lett. 115, 046603 (2015).

[36] C. Xu and M. G. Vavilov, Phys. Rev. B 95, 085139 (2017).

[37] I. Mondragon-Shem, A. Pal, T. L. Hughes, and C. R. Laumann, Phys. Rev. B 92, 064203 (2015).

[38] D. M. Kennes and C. Karrasch, Phys. Rev. B 93, 245129 (2016).

[39] X. Li, S. Ganeshan, J. H. Pixley, and S. Das Sarma, Phys. Rev. Lett. 115, 186601 (2015).

[40] T. Devakul and R. R. P. Singh, Phys. Rev. Lett. 115, 187201 (2015).

[41] W. De Roeck, F. Huveneers, M. Müller, and M. Schiulaz, Phys. Rev. B 93, 014203 (2016).

[42] L. Zhang, B. Zhao, T. Devakul, and D. A. Huse, Phys. Rev. B 93, 224201 (2016). 
[43] Following a recent proposal, such a behavior is not entirely unexpected, perhaps signalizing the breakdown of localization due to "hot bubbles" [41].

[44] T. C. Berkelbach and D. R. Reichman, Phys. Rev. B 81, 224429 (2010).

[45] S. Bera and A. Lakshminarayan, Phys. Rev. B 93, 134204 (2016).

[46] G. De Tomasi, S. Bera, J. H. Bardarson, and F. Pollmann, Phys. Rev. Lett. 118, 016804 (2017).

[47] Our definition of the discrete Fourier transform of $x_{n}$ : $y_{q}=\sum_{n=0}^{L-1} x_{n} e^{-l q n}, q=2 \pi a j / L$ and lattice spacing $a=1$.

[48] A. Weiße, G. Wellein, A. Alvermann, and H. Fehske, Rev. Mod. Phys. 78, 275 (2006).

[49] See Supplemental Material at http://link.aps.org/ supplemental/10.1103/PhysRevLett.118.196801 for additional data on return probability and various numerical convergence tests.

[50] R. Ketzmerick, K. Kruse, S. Kraut, and T. Geisel, Phys. Rev. Lett. 79, 1959 (1997).
[51] Notice that $\kappa_{\varepsilon}$ in Figs. 3(b1), 3(b3) exhibits small oscillations in $q$ that result from the finite system size.

[52] We would like to draw attention to a small additional feature that emerges for the high-energy kernel at very small wave numbers; as seen in Fig. 3(c3) with increasing time a cusp develops. It could be seen as a precursor indicating a stretched exponential shape in real space and the corresponding fit is shown in Fig. 3(a3). Its emergence at high energies first is understandable because of the relatively weak tendency to delocalization signalized by the observation $\Delta x_{\varepsilon}(t)<\Delta x_{\varepsilon}^{(0)}$.

[53] F. Evers and A. D. Mirlin, Rev. Mod. Phys. 80, 1355 (2008).

[54] M. Serbyn, Z. Papić, and D. A. Abanin, arXiv:1610 .02389

[55] I. García-Mata, O. Giraud, B. Georgeot, J. Martin, R. Dubertrand, and G. Lemarié, arXiv:1609.05857.

[56] K. S. Tikhonov, A. D. Mirlin, and M. A. Skvortsov, Phys. Rev. B 94, 220203 (2016). 